\title{
Space vector PWM technique for a three-level six-phase drive
}

\author{
E. A. R. Engku Ariff, O. Dordevic, M. Jones \\ Liverpool John Moores University, UK. e.a.engkuariff@2013.ljmu.ac.uk
}

Keywords: Multilevel inverters, multiphase drives, six-phase, space vector PWM, vector space decomposition.

\begin{abstract}
A space vector pulse-width modulation (SVPWM) strategy for a three-level six-phase drive based on vector space decomposition (VSD) approach is presented for the first time in this paper. The steps, requirements and conditions in determining the right switching sequences for the proposed modulation strategy are explained in detail. The developed SVPWM strategy is then simulated in Matlab/Simulink while driving a symmetrical six-phase induction machine running at no load. Obtained results prove the validity of the algorithm.
\end{abstract}

\section{Introduction}

Multilevel inverter supplied multiphase drives have been gaining the interest of researchers and industry in recent years. The use of power electronics means that the number of phases of the motor, $n$, is a degree of freedom allowing the current which flows through the motor to be shared between more phases, thus reducing the current rating of the power semiconductors used in the power converter [1]. Multiphase motors with $n$ equal to $5,6,7$, and 9 can be found in the literature. The machines with prime number of phases such as five- or seven-phase are rare in practice as they have to be custom made. Yet, a six- or nine-phase motor can be obtained by simply reconfiguring or by rewinding a standard threephase machine, which utilise the original frame of the threephase machine.

In addition, it is also possible to increase the number of inverter output voltage levels, $l$, using different power electronic converter topologies such as neutral-point clamped (NPC). The NPC inverter topology was proposed in-order to reduce the magnitude of harmonics which cause losses and pulsating torque in medium power drives while also allowing controllability of the output voltage fundamental [2]. As the number of inverter output voltage levels increases, the output voltage waveform approximates towards a sinusoidal waveform, thus, reducing the total harmonic distortion (THD) [3]. Furthermore, a multilevel topology can sustain a higher dc-link voltage compared to a two-level inverter using power semiconductors of the same rating. Therefore, it seems reasonable to increase both the number of motor phases and the number of inverter output voltage levels in order to achieve the benefits of both approaches. On the other hand, designing a suitable control algorithm for multiphase multilevel topologies is significantly more complex compared to the three-phase counterpart.
A multilevel multiphase drive was investigated for the first time in [4] using a three-level NPC supplied five-phase induction motor. Since then, several papers have been published discussing the modulation methods for multilevel multiphase drives, such as in [5-8]. However, the first successful implementation of SVPWM strategy for multilevel multiphase drive, based on the VSD approach of [9], is presented in [10]. In [11], the SVPWM strategy is optimised further and comparison between the optimised algorithm and carrier-based modulation is discussed. Meanwhile, in [12], the SVPWM strategy is adapted, optimised and applied to a three-level seven-phase inverter. This paper, for the first time, introduces an SVPWM strategy for a three-level symmetrical six-phase drive based on the VSD approach.

\section{Space vector algorithm}

In this paper, the aim of the space vector algorithm is to acquire a set of desired sinusoidal phase voltages at the inverter output. Reference phase voltages, $v_{p h}^{*}$, for a symmetrical six-phase drive are mutually shifted by $2 \pi / 6$, and are defined as:

$$
v_{p h}^{*}=V^{*} \cdot \cos [\omega t-2 \pi / 6(k-1)]
$$

where $k=1$ to 6 , which also corresponds to phases $a$ to $f$. The three-level symmetrical six-phase drive topology is shown in Figure 1. The relationship between the phase voltage, $v_{p h}$ and the leg voltage, $v_{L E G}$ can be derived, such that:

$$
v_{p h}=v_{L E G}-\frac{1}{6} \sum_{k=1}^{6} v_{L E G} .
$$

Note that, the second term of Equation (2) represents common mode voltage (CMV).

Considering a single leg of the inverter in Figure 1, one can see that there are four different combinations of switches (switches $S_{i}$ and $S_{i}^{\prime}$ are complementary switches). Possible

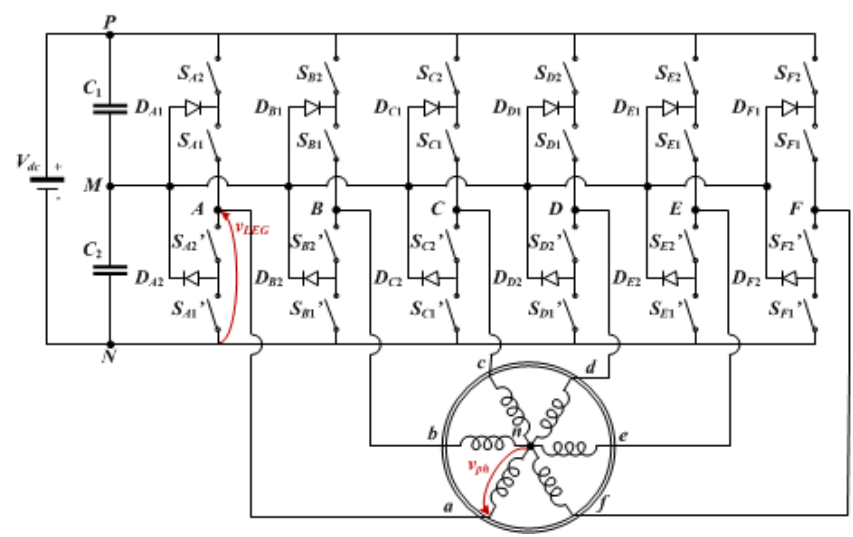

Figure 1: The three-level symmetrical six-phase drive. 
output leg voltage levels that can be obtained are $0, V_{d c} / 2, V_{d c}$ (referred to the dc-bus negative rail). The fourth combination produces high impedance output and is not considered in the algorithm. The voltage levels are further normalised by $V_{d c} / 2$ and denoted as 0,1 and 2 respectively. Therefore, the number of possible switching states for the analysed inverter is $3^{6}=$ 729 (0 to 728), which also can be represented in six-digit ternary numeral system as 000000 to 222222 , i.e. in normalised form for leg voltage levels.

\subsection{Three-level symmetrical six-phase space vectors}

The projection of space vectors (e.g. $v_{p h}$ or $v_{L E G}$ ) can be obtained using transformation matrix:

$\left[\begin{array}{c}v_{\alpha} \\ v_{\beta} \\ v_{x} \\ v_{y} \\ v_{0^{+}} \\ v_{0^{-}}\end{array}\right]=\frac{2}{6} \cdot\left[\begin{array}{cccccc}1 & \cos (\alpha) & \cos (2 \alpha) & \cos (3 \alpha) & \cos (4 \alpha) & \cos (5 \alpha) \\ 0 & \sin (\alpha) & \sin (2 \alpha) & \sin (3 \alpha) & \sin (4 \alpha) & \sin (5 \alpha) \\ 1 & \cos (2 \alpha) & \cos (4 \alpha) & \cos (6 \alpha) & \cos (8 \alpha) & \cos (10 \alpha) \\ 0 & \sin (2 \alpha) & \sin (4 \alpha) & \sin (6 \alpha) & \sin (8 \alpha) & \sin (10 \alpha) \\ 1 / 2 & 1 / 2 & 1 / 2 & 1 / 2 & 1 / 2 & 1 / 2 \\ 1 / 2 & -1 / 2 & 1 / 2 & -1 / 2 & 1 / 2 & -1 / 2\end{array}\right] \cdot\left[\begin{array}{c}v_{a} \\ v_{b} \\ v_{c} \\ v_{d} \\ v_{e} \\ v_{f}\end{array}\right]$

which is based on VSD approach, where $\alpha=2 \pi / 6$. The space vectors are projected into two mutually orthogonal twodimensional planes, $\alpha-\beta$ and $x-y$ and two zero-component axes, $0^{+}$and $0^{-}$. By applying Equation (1) into Equation (3), one finds that, the projection of $v_{p h}^{*}$ space vector in the $\alpha-\beta$ plane is travelling at angular speed of $\omega t$ and with magnitude of $V^{*}$, which forms a circle. On the contrary, the projections of $v_{p h}^{*}$ space vector in the $x-y$ plane, $0^{+}$and $0^{-}$axes are zero. The space vector projections of the obtained leg voltages (000000 to 222222 in normalised form) and phase voltages are discrete values.

The task of the SVPWM is to obtain the desired phase voltage $\left(v_{p h}^{*}\right)$, space vector by using discrete values of the obtained leg voltage $\left(v_{L E G}\right)$, space vectors, on average in each switching period, $T_{s}$. The projections of the leg and phase voltage space vectors into the $\alpha-\beta$ and $x-y$ plane as well as onto $0^{-}$axis are identical. The only difference is in the $0^{+}$axis, where the projection of the phase voltages is zero, while for the leg voltages it represents CMV. For this reason the task of SVPWM (to obtain $v_{p h}^{*}$ space vector by using $v_{L E G}$ space vectors) is possible, if the $0^{+}$axis is not considered. Hence the $0^{+}$axis i.e. $\mathrm{CMV}$, is not considered in the space vector modulation methods.

From the machine i.e. load perspective, the space vectors projected into the $\alpha-\beta$ plane are contributing to torque production in the machine. The space vectors projected into the $x-y$ plane contribute to the existence of low order harmonics which contribute to machine winding losses. Projections onto the $0^{-}$axis also contribute to the losses [13].

\subsection{Order-per-sector law}

Since the number of states is large, a method for reducing the possible switching states is implemented first. The order-persector law introduced in [10] reduces the number of switching states and simplifies the process of selecting the proper switching sequences. The principle of this method is simple and states that the obtained leg voltages i.e. switching states must follow the order of the reference phase voltages in each sector [10-12]. Phase voltages are sinusoidal waveforms, as in Equation (1), that change their mutual order at each $30^{\circ}$ i.e. $\pi / 6$ in one period. This angle also corresponds to the sector angle in the $\alpha-\beta$ plane. Thus, each possible switching state projected into the $\alpha-\beta$ plane is compared to the order of $v_{p h}^{*}$. The switching states which do not meet the requirement are discarded. For example, in the first sector (S1) i.e. $0 \leq \alpha<$ $\pi / 6$, the order of the phase voltages is $v_{a}^{*} \geq v_{b}^{*} \geq v_{f}^{*} \geq v_{c}^{*} \geq v_{e}^{*} \geq$ $v_{d}^{*}$. Switching state 120002 , which is projected into $\mathrm{S} 1$ in the $\alpha-\beta$ plane is discarded since its $v_{A}$ is less than its $v_{B}$, while the order-per-sector law requires the opposite. The implementation of this method significantly reduces the previous number of possible switching states, from 729 to 189 possible switching states. The remaining 189 possible switching states are applied into Equation (3) and their projections into $\alpha-\beta$ and $x-y$ planes as well as $0^{-}$axis are shown in Figure 2. Note that the projected switching states in Figure 2 are denoted with decimal representation. For obtaining switching state of each leg, conversion from decimal to ternary representation should be done.

\subsection{Determination of potential switching sequences}

In order to obtain sinusoidal output phase voltages, potential switching sequences are chosen such that, the average values of $x-y$ and $0^{-}$components are zero i.e. the low order harmonics corresponding to $x-y$ plane and $0^{-}$axis do not exist. Moreover, the number of the chosen space vectors ought to be the same as the number of phases of the machine, in this case, it is six [14]. In addition, to minimise losses and lessen $\mathrm{d} v / \mathrm{d} t$, the transition of output leg voltages also needs to be taken into consideration where the desirable level of transition is one, either increasing or decreasing in transition [15]. Also, it is desirable that the transition of the output leg voltages in one switching period is symmetrical i.e. in the first half of the switching period, the output leg voltages increase in transition level and in the other half, they decrease.

Besides meeting the previously mentioned requirements for obtaining the desired sinusoidal phase voltages, another important requirement, in determining the potential starting switching states for switching sequences selection, should also be met. The potential starting switching state should only consist either 'ones' or 'zeros', or combination of both when represented in six-digit ternary number [11]. This is because, in the first half of the switching period, the transition of leg voltages is increased by one level and it is impossible to choose starting switching state which has 'twos' since the maximum level of leg voltage is two. For example, among 28 possible switching states in S1, only seven can be chosen as the potential starting switching states (enclosed in red boxes) as shown in Figure 2(a).

Based on the identified potential starting switching states and the condition, where each leg voltage will increase its level by one in the first half of the switching period, one can count that there are 64 potential switching sequences in each of the twelve sectors. The possible transitions from space vectors for 
(a)
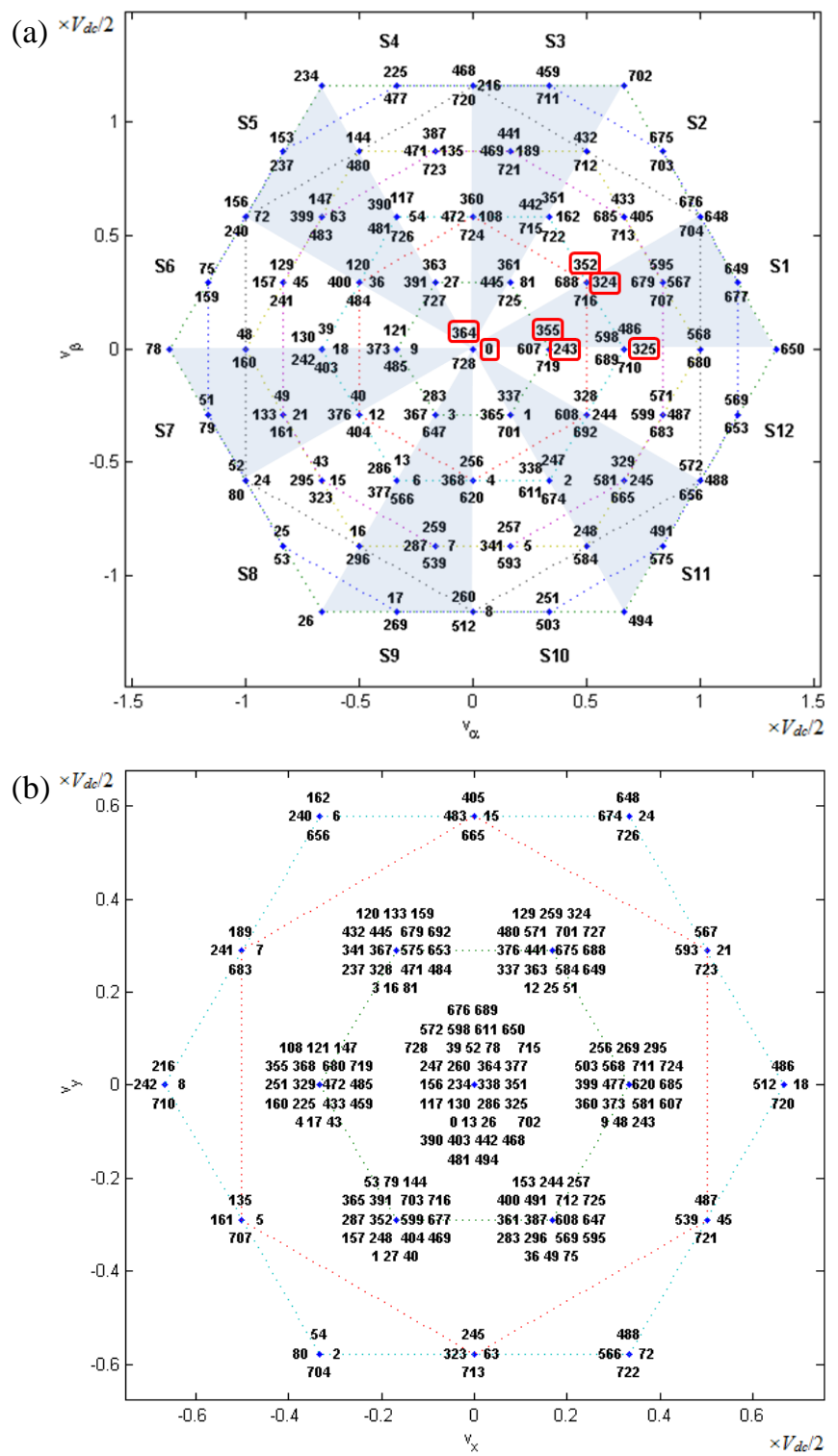

(c)

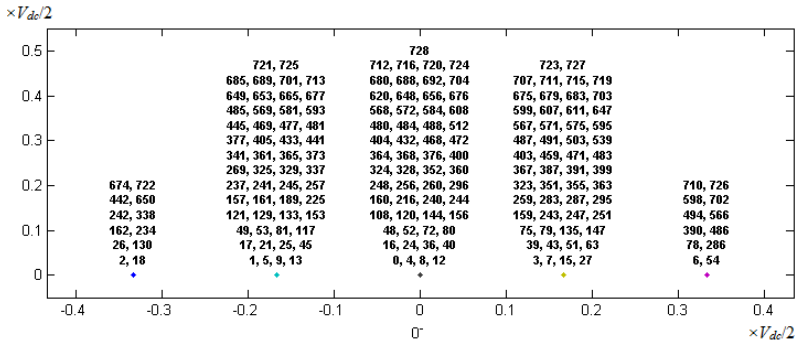

Figure 2: Projection of phase voltage space vectors of threelevel six-phase inverter after implementation of order-persector law in the: (a) $\alpha-\beta$ plane, (b) $x-y$ plane and (c) $0^{-}$axis.

S1 are illustrated with different colours of arrows as shown in Figure 3. Further, categorisation of 64 possible switching sequences can be done. One can find that they form only 32 unique transition patterns (each made of six different arrows in Figure 3). This is so, as each transition pattern contains several potential starting switching states, so the redundancy of switching sequences also exists. For example, in S1, the switching sequences 000000 - $100000-110000-110001$ $111001-111011-111111$ and $100000-110000-110001-$
111001 - 111011 - 111111 - 211111 are redundant and generate identical transition of space vectors in the $\alpha-\beta, x-y$ plane as well as on $0^{-}$axis.

Since the desired phase voltage $x-y$ and $0^{-}$components should be zero, selection process of choosing the right switching sequences can be done by conducting a graphical analysis of the transition patterns in the $x-y$ plane and $0^{-}$axis. In this way, by analysing the transition patterns for $\mathrm{S} 1$ in the $x-y$ plane, one can find that, only eight out of 32 transition patterns can provide voltage cancellation. The other 24 patterns do not surround the origin, hence cannot provide zero value on average in this plane. Subsequently, in $0^{-}$axis, another two transition patterns are identified such that the average value of $0^{-}$components cannot be zero. As a result, after the selection process, only six transition patterns remain per sector. Because of the switching sequence redundancy, there are 20 potential switching sequences that correspond to these six transition patterns, which should be considered further.

\subsection{Dwell time calculation and sector division}

The time duration of applied space vectors are called dwell times and can be calculated based on the volt-second balance principle and time balancing equation:

$$
\begin{gathered}
\underline{v}^{*} T_{s}=\underline{v}_{1} T_{1}+\underline{v}_{2} T_{2}+\underline{v}_{3} T_{3}+\underline{v}_{4} T_{4}+\underline{v}_{5} T_{5}+\underline{v}_{6} T_{6} \\
T_{s}=T_{1}+T_{2}+T_{3}+T_{4}+T_{5}+T_{6}
\end{gathered}
$$

Equations (4) and (5) can be rearranged in a matrix form as:

$$
\left[\begin{array}{c}
T_{1} \\
T_{2} \\
T_{3} \\
T_{4} \\
T_{5} \\
T_{6}
\end{array}\right]=\left[\begin{array}{cccccc}
v_{\alpha, 1} & v_{\alpha, 2} & v_{\alpha, 3} & v_{\alpha, 4} & v_{\alpha, 5} & v_{\alpha, 6} \\
v_{\beta, 1} & v_{\beta, 2} & v_{\beta, 3} & v_{\beta, 4} & v_{\beta, 5} & v_{\beta, 6} \\
v_{x, 1} & v_{x, 2} & v_{x, 3} & v_{x, 4} & v_{x, 5} & v_{x, 6} \\
v_{y, 1} & v_{y, 2} & v_{y, 3} & v_{y, 4} & v_{y, 5} & v_{y, 6} \\
1 & 1 & 1 & 1 & 1 & 1 \\
v_{0^{-}, 1} & v_{0^{-}, 2} & v_{0^{-}, 3} & v_{0^{-}, 4} & v_{0^{-}, 5} & v_{0^{-}, 6}
\end{array}\right]^{-1}\left[\begin{array}{c}
v_{\alpha}^{*} \\
v_{\beta}^{*} \\
v_{x}^{*} \\
v_{y}^{*} \\
1 \\
v_{0^{-}}^{*}
\end{array}\right] \cdot T_{s}
$$

The six-by-six matrix in Equation (6) contains space vector projections from Equation (3) for $\alpha, \beta, x, y$ and $0^{-}$axes of the six chosen space vectors. As mentioned, the $0^{+}$axis is not considered in the space vector algorithm, thus, the fifth row which corresponds to $0^{+}$axis is replaced by Equation (5). This will ensure that the sum of calculated dwell times $T_{1}$ to $T_{6}$, is $T_{\mathrm{s}}$. Since the desired output phase voltage is a sinusoidal waveform without any low order harmonics, the reference voltages for $x, y$ and $0^{-}$axes $\left(v_{x}^{*}, v_{y}^{*}\right.$ and $\left.v_{0-}^{*}\right)$ in Equation (6) are set to zero, while the reference voltages for $\alpha$, and $\beta$ axes $\left(v_{\alpha}^{*}\right.$, and $\left.v_{\beta}^{*}\right)$ are set to $V^{*} \cdot \cos (\omega t)$ and $V^{*} \cdot \sin (\omega t)$, respectively. Since the dwell times are calculated based on space vector projections, each of six transition patterns will have different values of dwell times. However, the redundant switching sequences which correspond to a particular transition pattern will have the same dwell times [12].

By placing constraint in Equation (6) that $0 \leq T_{i} \leq T_{s}$, (where $i=1, \ldots, 6)$ one finds that, the solutions for dwell time calculation for each transition pattern only exist in a certain region i.e. region of application in a sector. As a result, there are six sub-sectors per sector that correspond to each of six 
(a)

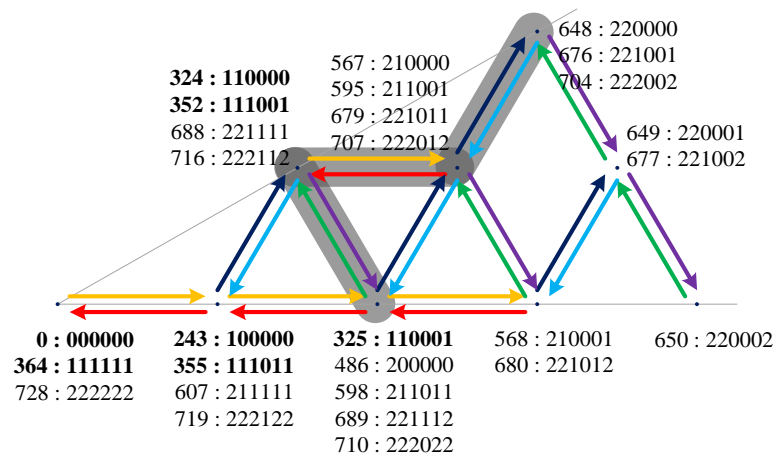

(b)

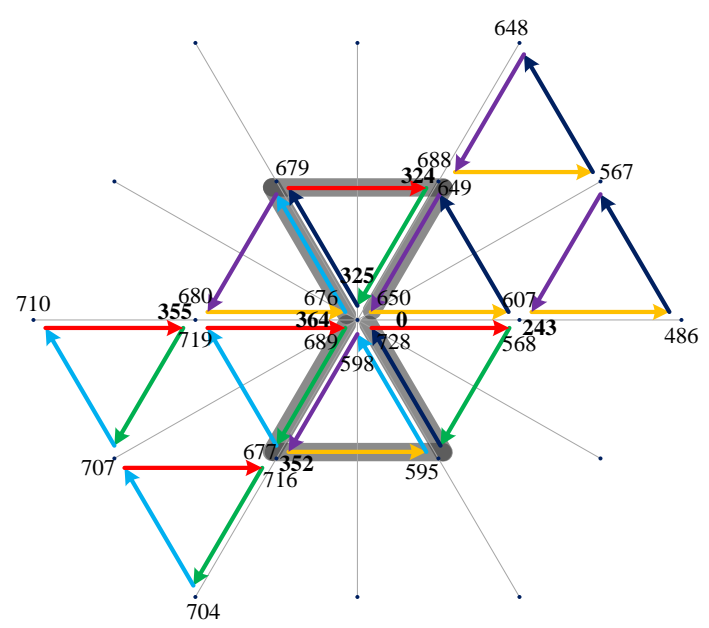

(c)

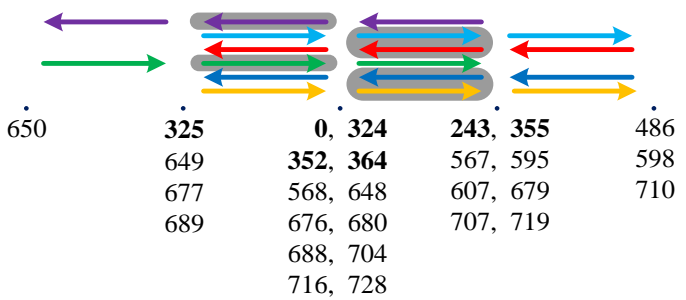

Figure 3: Possible transition of $v_{L E G}$ space vectors for the 1st sector in the: (a) $\alpha-\beta$ plane, (b) $x-y$ plane and (c) $0^{-}$axis.

transition patterns. The regions of application can be determined either by using analytical calculations [10] or by visualisation of numerical solutions [12]. In this paper, the latter method is adapted. The dwell times for all six transition patterns are repetitively calculated using Equation (6) with the constraints in place. The magnitude of the reference phase voltages, $V^{*}$ is gradually increased from zero to maximum value of $2 / 3 V_{d c}$, which is equivalent to the magnitude of the largest hexagon in the $\alpha-\beta$ plane. Since the analytical calculation is repetitively calculated, the determination of possible regions of application is implemented using a Matlab programme. The regions of application are visually determined by plotting a dot in the $\alpha-\beta$ plane if there are solutions for dwell times at that particular reference. For example, transition pattern enclosed in shaded region in Figure 3 forms a region of application shown with dots in Figure 4. The regions of application corresponding to each of six transition patterns in $\mathrm{S} 1$ (denoted by $A_{1}$ to $F_{1}$ ) are also shown in Figure 4. As it is indicated for S2, in Figure 4, all 12 sectors are partitioned into sub-sectors.

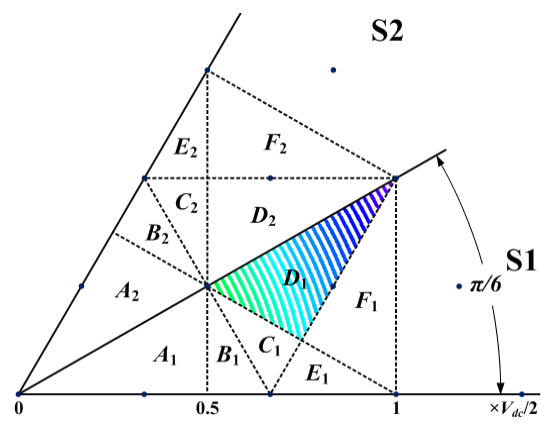

Figure 4: Regions of application in $\mathrm{S} 1$ and $\mathrm{S} 2$ (region of application for shaded transition pattern from Figure 3 is plotted by dots generated by Matlab code).

\subsection{Potential switching sequences optimisation}

The redundancy of the potential switching sequences for some transition patterns offers flexibility in selecting the right switching sequences. In order to select the right switching sequences, the leg voltage transitions between sub-sectors should be minimised, consequently minimising the switching losses [16]. Furthermore, it is desirable to have more than one output leg connected to the neutral point, $M_{p}$ i.e. leg output voltage is equal to $V_{d c} / 2$. This is because it offers more options in properly balancing the dc-link capacitors voltage [10]. Hence, one gets that, the selected switching sequences for S1 are as listed in Table 1. Note that, only switching states in the first half of the switching period are listed, since the order of the switching states is reversed in the second half.

\subsection{Sub-sectors determination}

Since the application of the sub-sectors i.e. selected switching sequences is dependent on the location of the projected $v_{p h}^{*}$ space vector, a proficient method on how to determine which sub-sectors should be applied as the projected $v_{p h}^{*}$ space vector is swapping through the sectors in the $\alpha-\beta$ plane, has to be implemented. One such method is described in [10] where the borders of the sub-sectors serve as limits in determining the location of the projected $v_{p h}^{*}$ space vector in the $\alpha-\beta$ plane at any given time. For example, an illustration of the method for $\mathrm{S} 1$ is shown in Figure 5. Relatively, there are five borders that divide the sub-sectors. These borders can be projected onto four perpendicular axes denoted with $P_{1}$ to $P_{4}$. The distances of these projected borders on the respective axes to the origin, $L_{1}$ to $L_{5}$ are calculated using simple trigonometric calculations based on Figure 4, which gives $L_{2}=L_{4}=0.25 V_{d c}, L_{3}=L_{1}$

\begin{tabular}{|c|c|}
\hline $\begin{array}{c}\text { Sub- } \\
\text { sector }\end{array}$ & Selected switching sequence \\
\hline$A_{1}$ & $110001-111001-111011-111111-211111-221111-221112$ \\
\hline$B_{1}$ & $110001-111001-111011-211011-211111-221111-221112$ \\
\hline$C_{1}$ & $110001-111001-211001-211011-221011-221111-221112$ \\
\hline$D_{1}$ & $110001-111001-211001-221001-221011-221111-221112$ \\
\hline$E_{1}$ & $110001-210001-211001-211011-221011-221012-221112$ \\
\hline$F_{1}$ & $110001-210001-211001-221001-221011-221012-221112$ \\
\hline
\end{tabular}

Table 1: Selected switching sequences for S1. 


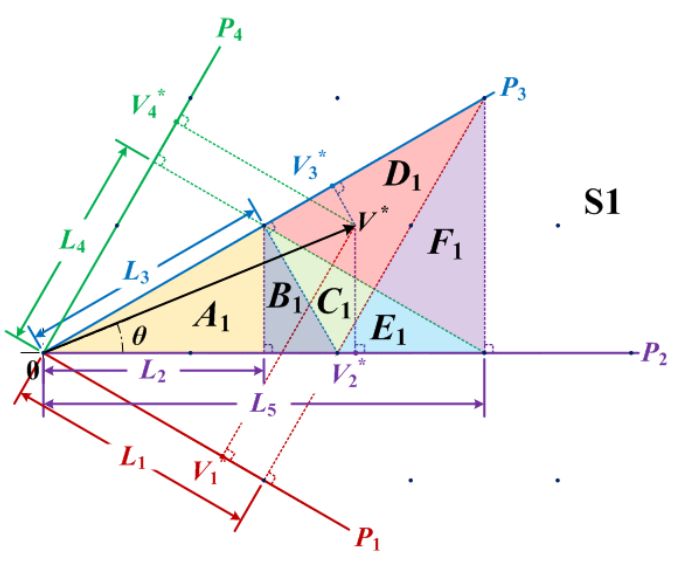

Figure 5: Sub-sectors determination based on the location of the projected $v_{p h}^{*}$ space vector in $\mathrm{S} 1$.

$=\sqrt{3} / 6 V_{d c}$ and $L_{5}=0.5 V_{d c}$ in length.

As can be seen in Figure 4, the sub-sectors of the even and odd sectors are mirrored to each other. As one might expect, for the second sector ( $\mathrm{S} 2)$, not only the four perpendicular axes, $P_{1}$ to $P_{4}$ and the projected borders on the respective axes are mirrored, but the length of the projected borders, $L_{1}$ to $L_{5}$ are also mirrored. Therefore, the conditions which define the location of the projected $v_{p h}^{*}$ space vector in determining the sub-sectors are the same for all sectors. These limits are then compared with the projections of projected $v_{p h}^{*}$ space vector onto the same corresponding axes, $P_{1}$ to $P_{4}$ which are denoted by $V_{1}^{*}$ to $V_{4}^{*}$. One finds that, by mapping the rest of the sectors onto $\mathrm{S} 1$ and $\mathrm{S} 2$, the projections of $v_{p h}^{*}$ space vector can be generalised as:

$$
\begin{array}{ll}
P_{i}=V^{*} \cdot \cos ((i-2) \pi / 6-(\theta-\pi / 6(k-1))) & \text { Oddsector } \\
P_{i}=V^{*} \cdot \cos ((i-2) \pi / 6-(k \pi / 6-\theta)) & \text { Evensector }
\end{array}
$$

where $k=1,3,5, \ldots, 11$ for odd sectors, $k=2,4,6, \ldots, 12$ for even sectors and $i=1,2,3,4$. A summary of the required conditions in determining the sub-sectors in all sectors are listed in Table 2.

\section{Simulation results}

The space vector PWM control scheme is developed and simulated in Matlab/Simulink as shown in Figure 6. Even though the steps explained for space vector algorithm in Section 2 are complex, the construction of the algorithm on the other hand, is quite simple. Since the sub-sectors are

\begin{tabular}{|c|l|}
\hline Sub-sector & \multicolumn{1}{|c|}{ Condition } \\
\hline$A_{k}$ & $V_{2}^{*} \leq L_{2}$ \\
\hline$B_{k}$ & $V_{2}^{*}>L_{2}, \quad V_{3}^{*} \leq L_{3}$ \\
\hline$C_{k}$ & $V_{3}^{*}>L_{3}, \quad V_{4}^{*} \leq L_{4}, \quad V_{1}^{*} \leq L_{1}$ \\
\hline$D_{k}$ & $V_{4}^{*}>L_{4}, \quad V_{1}^{*} \leq L_{1}$ \\
\hline$E_{k}$ & $V_{4}^{*} \leq L_{4}, \quad V_{1}^{*}>L_{1}$ \\
\hline$F_{k}$ & $V_{2}^{*} \leq L_{5}, \quad V_{4}^{*}>L_{4}, \quad V_{1}^{*}>L_{1}$ \\
\hline
\end{tabular}

Table 2: Conditions defining the sub-sectors that correspond to the location of the $v_{p h}^{*}$ space vector $(k=1,2, \ldots, 12)$.

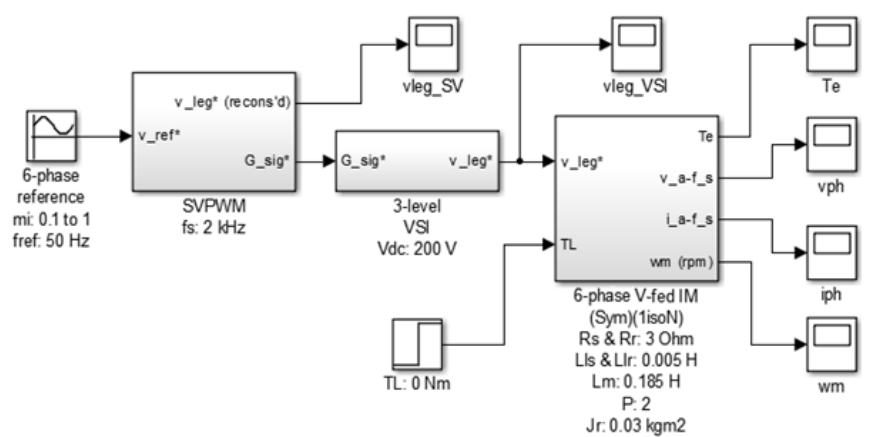

Figure 6: Simulation diagram of space vector PWM control scheme for three-level six-phase drive.

determined based on S1 and S2 for odd and even sectors, only switching sequences for those two sectors are needed i.e. stored as constants. Furthermore, in order to shorten the computation for dwell time calculation, the inverse matrices from Equation (6) corresponding to stored switching sequences are also stored as constants. The algorithm is tested in open loop, on the symmetrical six-phase induction machine operating with no load.

The simulation is run until the machine has reached steady state and the total harmonics distortion, THD is calculated for duration of one period for phase ' $a$ ' voltage and current, as:

$$
\mathrm{THD}_{v}=\sqrt{\sum_{k=2}^{h} v_{k}^{2} / v_{1}^{2}} \quad \operatorname{THD}_{i}=\sqrt{\sum_{k=2}^{h} i_{k}^{2} / i_{1}^{2}}
$$

The $v_{k}$ and $i_{k}$ represent the $k$-th components of voltage and current in the spectrum, while $v_{1}$ and $i_{1}$ are the fundamental values of phase voltage and current, respectively. Furthermore, harmonic components up to $h=420(21 \mathrm{kHz})$ i.e. first ten sidebands are included in both THD calculations. The THD is normally used in practice as a figure of merit in determining the performance of the modulation method [17].

Obtained simulation results of phase ' $a$ ' voltage and current THDs for full linear range of modulation index $\left(m_{i}\right)$ from 0.1 to 1 , with 0.05 increments, are shown in Figure 7 . As one might expect, the value of voltage and current THDs decreases as $m_{i}$ increases. This is because the number of output phase voltage levels also increases as $m_{i}$ increases which leads to the output phase voltage waveforms

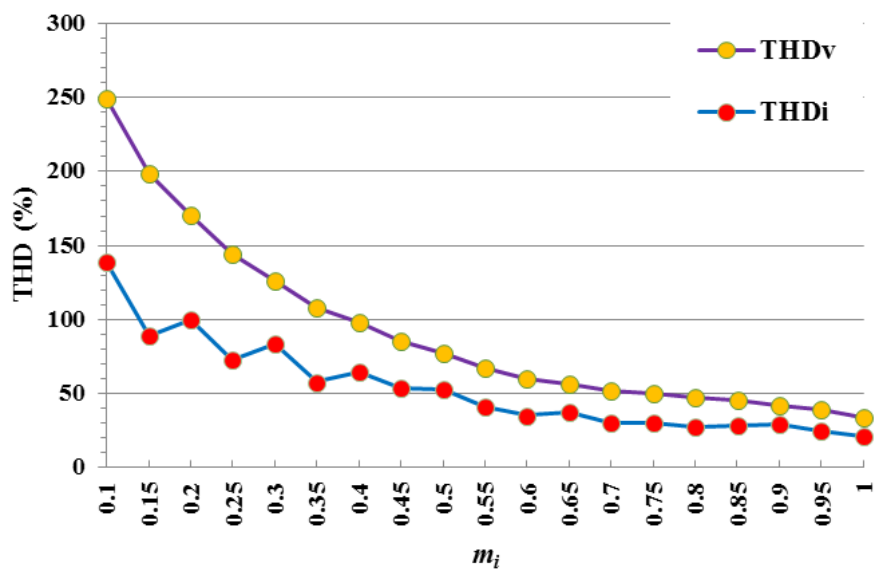

Figure 7: Phase ' $a$ ' voltage, $\mathrm{THD}_{v}$ and current, $\mathrm{THD}_{i}$ for $m_{i}$ ranging from 0.1 to 1 . 
approximate towards sinusoidal waveforms, thus reducing the THD.

Simulation results when $m_{i}=1$ are shown in Figure 8. The validity of the algorithm can be observed from the voltage waveforms. The desired value of the phase voltage fundamental $(100 \mathrm{~V})$, is obtained without existence of low order harmonics leading to low THD. Furthermore, the low order harmonics in the order of $6 k \pm 2(k=0,1,2,3 \ldots)$ and $3 k(k=1,3,5 \ldots)$ which are mapped into $x-y$ plane and $0^{-}$axis do not exist. A high current ripple can be noticed; however, it is purely the consequence of the low leakage inductance value $\left(L_{l s}=5 \mathrm{mH}\right.$, see Figure 6) which matches the machine available in the laboratory.

\section{Conclusion}

In this paper, an SVPWM strategy for three-level six-phase inverter based on VSD approach is presented for the first time. A method for reducing the number of possible switching states is implemented. Afterwards, the right switching sequences are determined such that the average values for $x-y$ and $0^{-}$components are zero. The dwell times corresponding to each sub-sector are calculated and implemented based on the location of the applied reference phase voltages. The developed SVPWM strategy is simulated for a symmetrical six-phase induction machine running at no load using Matlab/Simulink. The presented simulation results show that the obtained output phase voltages and currents do not contain low order harmonics and thus validate the proposed algorithm.
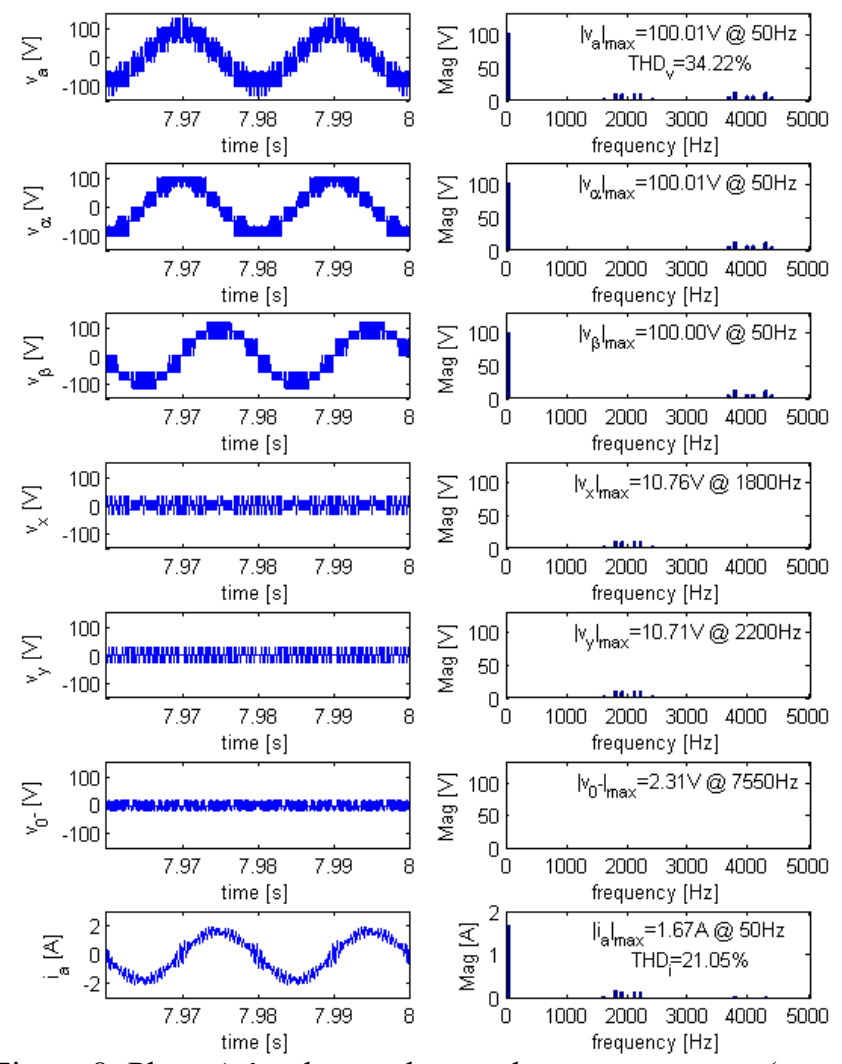

Figure 8: Phase ' $a$ ' voltage, phase voltage components $\left(v_{\alpha}, v_{\beta}\right.$, $v_{x}, v_{y}$ and $\left.v_{0-}\right)$, phase ' $a$ ' current and corresponding spectra.

\section{References}

[1] E. Levi, "Advances in converter control and innovative exploitation of additional degrees of freedom for multiphase machines," IEEE Transactions on Industrial Electronics, vol. 63, pp. 433-448, 2016.

[2] A. Nabae, I. Takahashi, and H. Akagi, "A new neutral-pointclamped PWM inverter," IEEE Transactions on Industry Applications, vol. 17, pp. 518-523, 1981.

[3] L. M. Tolbert, F. Z. Peng, and T. G. Habetler, "Multilevel converters for large electric drives," IEEE Transactions on Industry Applications, vol. 35, pp. 36-44, 1999.

[4] S. Lu and K. Corzine, "Multilevel multi-phase propulsion drives," in IEEE Electric Ship Technologies Symposium, 2005, pp. 363-370.

[5] J. S. Hu, K. Y. Chen, T. Y. Shen, and C.-H. Tang, "Analytical solutions of multilevel space-vector PWM for multiphase voltage source inverters," IEEE Transactions on Power Electronics, vol. 26, pp. 1489-1502, 2011.

[6] J. I. Leon, O. Lopez, L. G. Franquelo, J. Doval-Gandoy, S. Vazquez, J. Alvarez, "Multilevel multiphase feedforward space-vector modulation technique," IEEE Transactions on Industrial Electronics, vol. 57, pp. 2066-2075, 2010.

[7] O. Lopez, J. Alvarez, J. Doval-Gandoy, and F. D. Freijedo, "Multilevel multiphase space vector PWM algorithm," IEEE Transactions on Industrial Electronics, vol. 55, pp. 1933-1942, 2008.

[8] S. Qingguo, Z. Xiaofeng, Y. Fei, and Z. Chengsheng, "Research on space vector PWM of five-phase three-level inverter," in International Conference on Electrical Machines and Systems, ICEMS, 2005, pp. 1418-1421.

[9] Y. Zhao and T. A. Lipo, "Space vector PWM control of dual three-phase induction machine using vector space decomposition," IEEE Transactions on Industry Applications, vol. 31, pp. 1100-1109, 1995.

[10] L. Gao and J. E. Fletcher, "A space vector switching strategy for three-level five-phase inverter drives," IEEE Transactions on Industrial Electronics, vol. 57, pp. 2332-2343, 2010.

[11] O. Dordevic, M. Jones, and E. Levi, "A comparison of carrierbased and space vector PWM techniques for three-level fivephase voltage source inverters," IEEE Transactions on Industrial Informatics, vol. 9, pp. 609-619, 2013.

[12] O. Dordevic, E. Levi, and M. Jones, "A vector space decomposition based space vector PWM algorithm for a threelevel seven-phase voltage source inverter," IEEE Transactions on Power Electronics, vol. 28, pp. 637-649, 2013.

[13] D. Dujic, A. Iqbal, and E. Levi, "A space vector PWM technique for symmetrical six-phase voltage source inverters," in European Power Electronics And Adjustable Speed Drives Conference, EPE, Aalborg, Denmark, 2007, pp. 24-32.

[14] J. W. Kelly, E. G. Strangas, and J. M. Miller, "Multiphase space vector pulse width modulation," IEEE Transactions on Energy Conversion, vol. 18, pp. 259-264, 2003.

[15] H. W. van der Broeck, H. C. Skudelny, and G. V. Stanke, "Analysis and realization of a pulsewidth modulator based on voltage space vectors," IEEE Transactions on Industry Applications, vol. 24, pp. 142-150, 1988.

[16] B. P. McGrath, D. G. Holmes, and T. Lipo, "Optimized space vector switching sequences for multilevel inverters," IEEE Transactions on Power Electronics, vol. 18, pp. 1293-1301, 2003.

[17] O. Dordevic, M. Jones, and E. Levi, "Analytical formulas for phase voltage RMS squared and THD in PWM multiphase systems," IEEE Transactions on Power Electronics, vol. 30, pp. 1645-1656, 2015. 